\title{
Mitarbeit des ALTS an einer Webseite - initiiert von der Fachgruppe Lebensmittelmikrobiologie und -hygiene der DGHM
}

\author{
Dr. M. Ludewig \\ Universität Leipzig, Institut für Lebensmittelhygiene, An den Tierkliniken 1, D-04103 Leipzig
}

\section{Zusammenfassung/Erläuterung}

Auf dem diesjährigen 8. Fachsymposium der Fachgruppe Lebensmittelmikrobiologie und -hygiene der DGHM in Zusammenarbeit mit der Fachgruppe der VAAM in Suhl wurde als Ergebnis einer Podiumsdiskussion mit dem Arbeitstitel „Wo steht die Lebensmittelmikrobiologie in Deutschland heute?“ die Gründung einer Internetseite beschlossen.

Anliegen der Initiative soll die Gründung einer Plattform sein, in der alle Fachbereiche, die insbesondere auf dem Gebiet der Lebensmittelmikrobiologie aber auch in anderen lebensmittelhygienischen Arbeitsgebieten tätig sind, zusammengeführt werden. Ausdrücklich wurde von den Initiatoren darauf verwiesen, dass alle Fachbereiche mit ihren Querverbindungen zur Mitgestaltung aufgerufen sind.

Die Ziele dieser Internetseite wurden bisher nur angedacht. Folgende erste Ziele wurden formuliert: (a) Veröffentlichungen von mikrobiologischen Richtwerten, (b) Veröffentlichung von Informationen zur Beurteilung von Lebensmitteln, (c) Vorstellen von Methoden und (d) Plattform zur Diskussion und Meinungsaustausch.
Nach Rücksprache mit Herrn Professor Holzapfel wäre eine Mitarbeit des ALTS sehr willkommen.

Die Initiatoren für die Ausgestaltung der Webseite sind Herr Professor Dr. J. Krämer (Landwirtschaftliche- und Lebensmittelmikrobiologie, Universität Bonn), Herr Professor Dr. W. Holzapfel (Institut für Hygiene und Toxikologie Bundesforschungsanstalt für Ernährung und Lebensmittel - Standort Karlsruhe) und Herr Professor Dr. H. Schmidt (Institut für Lebensmitteltechnologie, FG Lebensmittelmikrobiologie, Universität Hohenheim).

\section{Diskussionsergebnis}

Der ALTS erachtet eine Mitarbeit an der dargestellten Web-Seite der DGHM als sinnvoll und empfiehlt seinen Mitgliedern, sich bei Interesse mit Frau Dr. Ludewig (Universität Leipzig) in Verbindung zu setzen. 